\title{
Gallbladder carcinoma: an initial clinical experience of reduced field-of-view diffusion-weighted MRI
}

\author{
Sisi Wu, Xianlun Zou, Qiuxia Wang, Daoyu Hu, Zhen Li and Chuou Xu* (D)
}

\begin{abstract}
Background: The purpose of this study is to compare the diagnostic value, imaging quality and apparent diffusion coefficient (ADC) value of reduced field-of-view diffusion-weight imaging (r-FOV DWI) and full field-of-view diffusion-weight imaging (f-FOV DWI) in patients with gallbladder carcinoma and other lesions of gallbladder.

Methods: Two hundred ninety-six patients with gallbladder diseases underwent both r-FOV DWI and f-FOV DWI on a 3.0 T MRI scanner. Two radiologists assessed subjective image quality parameters independently. The Wilcoxon signed-rank test was used to compare subjective qualitative image score. Objective quality values and the mean $A D C$ values were analyzed by paired $t$-test. The correlation between pathological results and mean ADC value were estimated using Spearman rank correlation analysis.

Results: The CNR value $(10.23 \pm 2.92)$ and image quality score $(13.84 \pm 1.07)$ of $r$-FOV DWI were significantly higher than those of $\mathrm{f}$-FOV DWI $(5.24 \pm 1.29 P<0.001 ; 10.41 \pm 1.11 P<0.001)$. There was no significant difference between mean ADC values of the two DWI sequences for all three groups (Group 1, chronic cholecystitis; Group 2, benign lesions of gallbladder; Group 3, gallbladder carcinoma. $P=0.239,0.974$ and 0.226 respectively). For both DWI sequences, the mean $A D C$ values were the highest in the group of cholecystitis and the lowest in the group of gallbladder carcinoma ( $2.49 \pm 0.14$ vs $1.49 \pm 0.12 ; 2.50 \pm 0.14$ vs $1.50 \pm 0.13$, for f-FOV and $r$-FOV respectively), the differences among groups were statistically significant $(P<0.01)$. The mean ADC values for both DWI sequences were negatively correlated with the group number, which increased with the malignant tendency of lesions $(r=-$ $0.892, P<0.01 ; r=-0.913, P<0.01$ for $f-F O V$ and $r$-FOV respectively).
\end{abstract}

Conclusion: Reduced Field-of-view Diffusion-weighted MRI is a good tool to diagnosis the gallbladder carcinoma, with better image quality and without affecting ADC values.

Keywords: Diffusion-weighted imaging, Full field-of-view, Gallbladder carcinoma, Image quality, Reduced field-ofview

\footnotetext{
* Correspondence: xchuou@163.com

Department of Radiology, Tongji Hospital, Tongji Medical College, Huazhong University of Science and Technology, 1095 Jiefang Avenue, Qiaokou district, Wuhan 430030, Hubei, China
}

C C The Author(s). 2020 Open Access This article is licensed under a Creative Commons Attribution 4.0 International License, which permits use, sharing, adaptation, distribution and reproduction in any medium or format, as long as you give appropriate credit to the original author(s) and the source, provide a link to the Creative Commons licence, and indicate if changes were made. The images or other third party material in this article are included in the article's Creative Commons licence, unless indicated otherwise in a credit line to the material. If material is not included in the article's Creative Commons licence and your intended use is not permitted by statutory regulation or exceeds the permitted use, you will need to obtain permission directly from the copyright holder. To view a copy of this licence, visit http://creativecommons.org/licenses/by/4.0/ The Creative Commons Public Domain Dedication waiver (http://creativecommons.org/publicdomain/zero/1.0/) applies to the data made available in this article, unless otherwise stated in a credit line to the data. 


\section{Background}

Primary gallbladder carcinoma is a common malignant tumor of the biliary system with high lethal malignancy and poor prognosis. Most cases of gallbladder carcinoma are diagnosed postoperatively, which are called occult gallbladder carcinoma. Early detection and treatment of gallbladder cancer can improve the prognosis. Furthermore some benign lesions, such as gallbladder adenoma and gallbladder polyps, have risk of developing malignancy and also require early detection and treatment [1]. However, a reported 5-year survival rate of advanced gallbladder cancer is merely 3\% [2]. Therefore, early detection and differentiation benign lesions from malignant lesions for gallbladder are of great significance for clinical treatment.

Over the past few decades, ultrasonography was considered as one of the most important imaging modality for detection of gallbladder diseases. However, it is limited in the diagnosis of early lesion because of the lower sensitivity and specificity [3]. Recently, diffusion weighted imaging (DWI) has been reported to facilitate the diagnosis of gallbladder cancer, and it can also differentiate gallbladder benign and malignant disorders [46]. The apparent diffusion coefficient (ADC) value of DWI can indicate the histological grade of primary gallbladder carcinoma [7].

Currently, the standard sequence used for clinical DWI is full filed-of-view (f-FOV) single-shot echo-planar imaging (SS-EPI). Due to its long readout time and low bandwidth in the phase-encode direction, SS-EPI is prone to artifacts, distortion and blurring, which affects the accuracy of the detection of small lesions [8]. The reduced field-of-view (r-FOV) DWI using two-dimensional spatially selective excitation and a $180^{\circ}$ refocusing pulse to reduce the FOV in the phase-encode direction, not only provides fewer artifacts, but also higher quality images [9]. This technique has been widely used in spinal cord, rectum, urinary bladder and prostate [8-11]. However, to the best of our knowledge, no previous studies have been reported in the detection of gallbladder diseases using r-FOV DWI. The purpose of this study is to compare the diagnostic value, imaging quality and apparent diffusion coefficient (ADC) value of reduced field-of-view diffusion-weight imaging (r-FOV DWI) and full field-of-view diffusion-weight imaging (f-FOV DWI) in patients with gallbladder carcinoma and other lesions of gallbladder, and also to determine whether the ADC values of gallbladder lesions for both DWI sequences are correlated with the pathological results.

\section{Material and methods}

\section{Patients}

This retrospective study was approved by the Institutional Review Board of our institution and requirement for written informed consent was waived. Between November 2016 and January 2019, 296 patients were enrolled in this study, the inclusion criteria were employed as follows: 1) all patients having pathological results of gallbladder lesions; 2) patients underwent pretreatment MRI for both f-FOV and r-FOV DWI imaging. And the exclusion criteria were: 1) patients receiving previous surgery or medical treatment; 2) patients underwent MR scanning with inconsistent scan parameters. Among the 296 patients, 45 patients were excluded from this study because of previous surgery or medical treatment, 190 patients were excluded due to inconsistent MR scan parameters. As a result, 61 patients were included in this study ( 28 males; 33 females; mean age $55.80 \pm 11.63$ years; range $25-78$ years). All the lesions were confirmed by pathological results, including 22 cases of chronic cholecystitis, 22 cases of gallbladder carcinoma, 7 cases of gallbladder adenomyomatosis, 7 cases of gallbladder adenoma and 3 cases of gallbladder polyps (Fig. 1).

\section{MRI data acquisition}

All patients underwent magnetic resonance imaging (MRI) studies on a GE $3.0 \mathrm{~T}$ Discovery 750 scanner with a 32-channel torso phase-array coil. All patients fasted for at least $6 \mathrm{~h}$ and had breathing training before scanning. Breath gating was employed during MRI scanning.

The diffusion-weighted SS-EPI f-FOV and r-FOV images were acquired in the axial plane, focusing on lesions. The scanning parameters of the SS-EPI DWI (fFOV DWI) were as follows: $\mathrm{TR}=3157 \mathrm{~ms}$, $\mathrm{TE}=57.5 \mathrm{~ms}$, FOV $=36 \mathrm{~mm} \times 28.8 \mathrm{~mm}$, matrix $=160 \times 128$, slice thickness $=4 \mathrm{~mm}$, gap $=1 \mathrm{~mm}$, bandwidth $= \pm 250 \mathrm{KHz}, \mathrm{NEX}=$ 10 , and $\mathrm{b}=0$ and $800 \mathrm{~s} / \mathrm{mm} 2$. The scanning parameters of r-FOV DWI were as follows: $\mathrm{TR}=3000 \mathrm{~ms}$, $\mathrm{TE}=55.3$ $\mathrm{ms}, \quad F O V=20 \mathrm{~mm} \times 10 \mathrm{~mm}, \quad$ matrix $=128 \times 64$, slice thickness $=4 \mathrm{~mm}, \quad$ gap $=1 \mathrm{~mm}, \quad$ bandwidth $= \pm 250 \mathrm{KHz}$, $\mathrm{NEX}=10$, and $\mathrm{b}=0$ and $800 \mathrm{~s} / \mathrm{mm}^{2}$ (Table 1 ).

\section{Image analysis Objective analysis}

All the images were reviewed and analyzed on picture archiving and communication system (PACS) and GE workstation 4.6. The r-FOV and f-FOV images were blindly assessed by two independent radiologists with 6 years and 10 years of imaging experience, signal intensity, background signal intensity, and intercostals signal intensity were measured and calculated. The signal-to-noise ratio (SNR) and cintrast-to-noise (CNR) were calculated as the following formulas: 


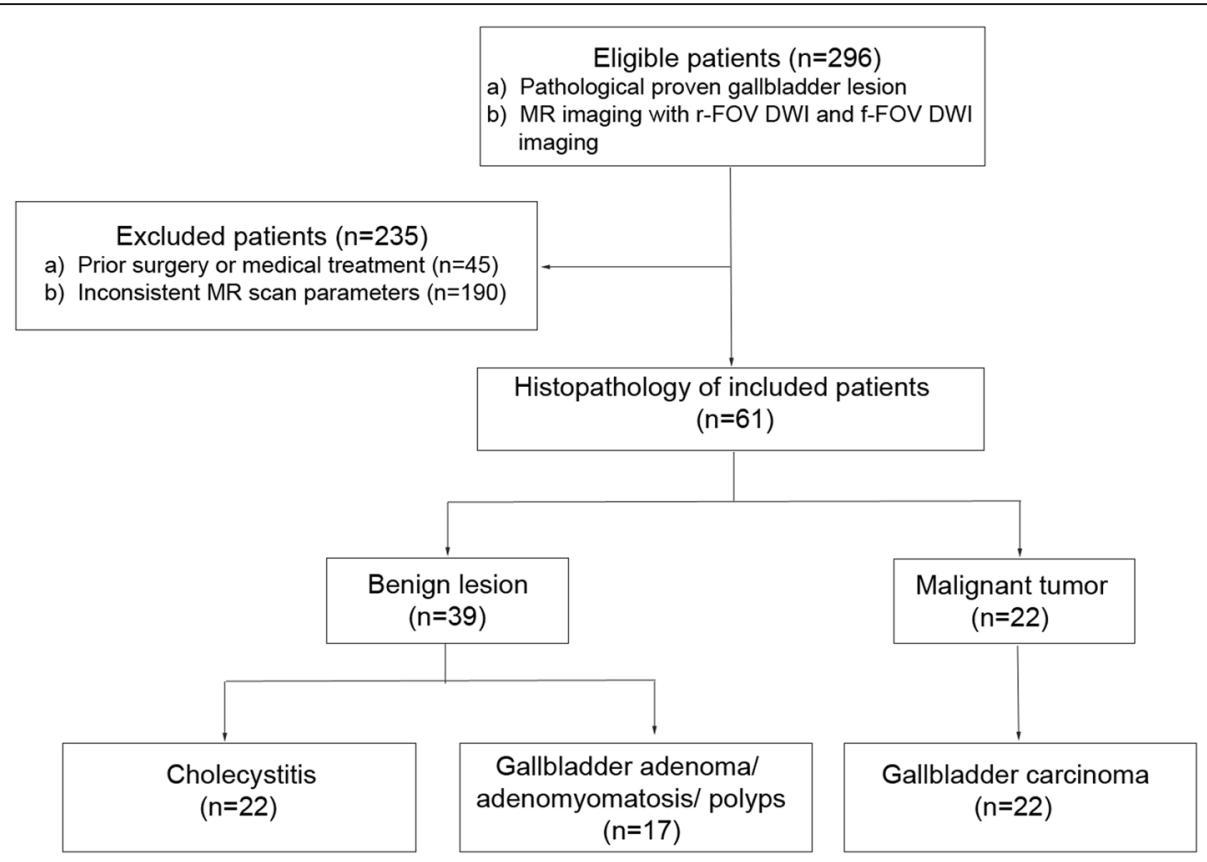

Fig. 1 Flow diagram demonstrating patient and tumor characteristics

$$
\frac{\mathrm{SNR}=\frac{\mathrm{S}_{\text {lesion }}}{\mathrm{SD}_{\text {background }}} \mathrm{CNR}=\underline{\left|\mathrm{S}_{\text {lesion }}-\mathrm{S}_{\text {tissue }}\right|}}{\sqrt{\mathrm{SD}_{\text {lesion }}{ }^{2}+\mathrm{SD}_{\text {tissue }}}{ }^{2}}
$$

The SNR was calculated as the ratio between the mean signal intensity inside the lesion $\left(\mathrm{S}_{\text {lesion }}\right)$ and the standard deviation of background noise ( $\left.\mathrm{SD}_{\text {background }}\right)$; The $\mathrm{CNR}$ was defined as the ratio of the mean signal intensity difference between lesion $\left(\mathrm{S}_{\text {lesion }}\right)$ and normal tissue $\left(\mathrm{S}_{\text {tissue }}\right)$ divided by the standard deviation of the lesion $\left(\mathrm{SD}_{\text {lesion }}\right)$ and normal tissue $\left(\mathrm{SD}_{\text {tissue }}\right)$.

All of the ADC values were calculated on the GE workstation 4.6 with a standard software package. The ADC value of a lesion was measured by carefully drawing a region of interest (ROI) around the largest area of the lesion for adjacent three sections $(b=800 \mathrm{~s} /$ $\mathrm{mm}^{2}$ ) with homogenous signal intensity, without

Table 1 Imaging Parameters for f-FOV and r-FOV DiffusionWeighted Imaging

\begin{tabular}{lll}
\hline Sequence parameter & f-FOV & r-FOV \\
\hline TR/TE (msec) & $3157 / 57.5$ & $3000 / 55.3$ \\
Thickness/Gap (mm) & $4 / 1$ & $4 / 1$ \\
FOV (mm) & $360 \times 288$ & $200 \times 100$ \\
Matrix & $160 \times 128$ & $128 \times 64$ \\
NEX & 10 & 10 \\
Bandwidth (KHZ) & 250 & 250 \\
b-value (sec/mm $\left.{ }^{2}\right)$ & 0,800 & 0,800 \\
\hline
\end{tabular}

$T R$ repetition time, TE echo time, FOV field of view, $f$-FOV full FOV, $r$-FOV reduced FOV, NEX number of excitations artifacts or deformations. The lesion was identified on $\mathrm{b}=0 \mathrm{~s} / \mathrm{mm}^{2}$. Areas containing bile, blood, cystic degeneration or necrosis were avoided. This procedure was performed 3 times for each patient by a single observer. The average of 3 measurements was taken as the average $\mathrm{ADC}$ value.

\section{Subjective analysis}

The r-FOV DWI and f-FOV DWI images were evaluated by two experienced radiologists based on the following characteristics: sharpness, distortion, artifacts and lesion conspicuity, scoring was based on a 4-point scale as follows: sharpness $(1=$ poor, $2=$ fair, $3=$ good, $4=$ excel lent); distortion $(1=$ severe distortion, $2=$ moderate distortion, $3=$ slight distortion, $4=$ no distortion); artifacts $(1=$ severe artifacts, may interfere the diagnostic information, 2 = severe artifacts, may partially interfere the diagnostic information, $3=$ slight artifacts, no interfere the diagnostic information, $4=$ no artifacts); lesion conspicuity $(1=$ poor, considered unrecognized, $2=$ fair, most of the outlines unclear, $3=$ good, small part of outline unclear, 4 =excellent, clear outline). Total subjective image quality score was calculated by adding the above four values all together in the same imaging section.

\section{Statistical analysis}

All analyses were performed using SPSS for Windows, version 19.0 (IBM, Armonk, NY). The interobserver variability of objectively rated image quality (including CNR and SNR) was assessed using the intraclass 
correlation coefficient (ICC) test $(0.00-0.20$, poor agreement; $0.21-0.40$, fair agreement; $0.41-0.60$, moderate agreement; $0.61-0.80$, good agreement; $0.81-1.00$, excellent agreement). The interobserver variability of subjective image quality score (including sharpness, distortion, artifacts and lesion conspicuity) was evaluated by weighted kappa statistics $(0.00-0.20$, poor agreement; $0.21-0.40$, fair agreement; $0.41-0.60$, moderate agreement; $0.61-0.80$, good agreement; $0.81-$ 1.00 , excellent agreement). The Wilcoxon signed-rank test was used to compare the subjective qualitative image scores (sharpness, distortion, artifacts and lesion conspicuity) between $\mathrm{r}$-FOV DWI and f-FOV DWI. Objective quality values (SNR, CNR) and the mean ADC values were statistically analyzed by paired $t$-test for the two DWI sequences, and the correlation between pathological results and mean ADC value was estimated using Spearman rank correlation analysis. Results with a $P$ value less than 0.05 were considered statistically significant.

\section{Results}

1. Pathological Results

Of all 61 patients, the pathological results revealed 22 cases $(36.06 \%)$ of gallbladder carcinoma, 7 cases $(11.48 \%)$ of gallbladder adenoma, 7 cases $(11.48 \%)$ of gallbladder adenomyomatosis, 3 cases (4.92\%) of gallbladder polyps and 22 cases $(36.06 \%)$ of cholecystitis. These cases were divided into 3 groups according to the malignant tendency of the lesion (Group 1, chronic cholecystitis; Group 2, benign lesions of gallbladder including gallbladder adenomyomatosis, gallbladder adenoma and gallbladder polyps; Group 3, gallbladder carcinoma), the larger the group number was, the more likely the lesion tends to be malignant.

2. Interobserver Variability of Image Quality Objective image quality values (SNR, CNR) of both DWI methods had good to excellent agreement. The ICC values ranged from 0.776 to 0.967 . All of the subjective image quality scores (sharpness, distortion, artifacts and lesion conspicuity) had good to excellent agreement, the $\mathrm{K}$ values between two radiologists ranged from 0.769 to 0.873 (Table 2).

3. Comparison of Image Quality

Based on the formula of image resolution $=\mathrm{FOV} /$ matrix, the image resolution of f-FOV DWI and r-FOV DWI was $2.25 \times 2.25 \mathrm{~mm}$ and $1.56 \times 1.56$ $\mathrm{mm}$ respectively. The comparison of image quality scores assessed by two radiologists between $\mathrm{f}$-FOV and r-FOV DWI is shown in
Table 3. The CNR value of r-FOV DWI was significantly higher than that of f-FOV DWI $(10.23 \pm 2.92,5.24 \pm 1.29, \boldsymbol{P}<0.001)$ while the SNR value of $r$-FOV DWI was lower than that of $f$ FOV DWI. The subjective image quality scores of sharpness (3.43 $\pm 0.47,2.64 \pm 0.59, \boldsymbol{P}<0.001)$, distortion $(3.35 \pm 0.61,2.59 \pm 0.54, \boldsymbol{P}<0.001)$, artifacts (3.66 $\pm 0.46,2.75 \pm 0.54, \boldsymbol{P}<0.001)$, lesion conspicuity $(3.39 \pm 0.55,2.48 \pm 0.62, \boldsymbol{P}<0.001)$ and total subjective image quality score $(13.84 \pm 1.07$, $10.41 \pm 1.11, \boldsymbol{P}<0.001)$ for r-FOV DWI were significantly higher than those for f-FOV DWI (Fig. 2).

4. Quantitative Assessment of ADC values of Gallbladder lesions

For both DWI sequences, the mean ADC values in the group of cholecystitis were the highest $(2.49 \pm 0.14$, $2.50 \pm 0.14$, respectively) while the mean ADC values in the group of malignant tumor were the lowest $(1.49 \pm$ $0.12,1.50 \pm 0.13$, respectively), the differences among groups were statistically significant $(P<0.01)$ (Fig. 3). There was no significant difference between mean ADC values of f-FOV DWI and r-FOV DWI $(P=0.625)$. There was no significant difference between mean ADC values of the two DWI sequences for cholecystitis, benign lesions or malignant tumor either $(P=0.239,0.974$ and 0.226 respectively). The ADC values of different pathological results of gallbladder lesions in the two DWI sequences are shown in Table 4. The mean ADC values for both DWI sequences were negatively correlated with the group number, which increased with the malignant tendency of lesions $(r=-0.892, \mathrm{P}<0.01 ; \mathrm{r}=-$ $0.913, \mathrm{P}<0.01$ respectively) (Table 5 ).

\section{Discussion}

Diffusion-Weighted imaging (DWI) is a non-invasive imaging technique which reflects changes in tissue cell density and microstructure [12]. Single-shot echoplanar imaging (SSEPI) technique was employed in

Table 2 Interobserver Variability of Image Quality of f-FOV and r-FOV Diffusion-Weighted Imaging

\begin{tabular}{lll}
\hline Image parameter & f-FOV & r-FOV \\
\hline SNR & $0.967(0.946-0.980)$ & $0.938(0.899-0.962)$ \\
CNR & $0.776(0.652-0.859)$ & $0.878(0.804-0.925)$ \\
Sharpness & $0.769(0.606-0.908)$ & $0.833(0.689-0.965)$ \\
Distortion & $0.873(0.751-0.969)$ & $0.799(0.652-0.920)$ \\
Artifacts & $0.837(0.686-0.966)$ & $0.855(0.691-0.967)$ \\
Lesion conspicuity & $0.826(0.682-0.942)$ & $0.846(0.711-0.968)$ \\
\hline
\end{tabular}

Data in parentheses are $95 \%$ confidence intervals, SNR = signal-to-noise ratio, $\mathrm{CNR}=$ contrast-to-noise ratio 
Table 3 Comparison of Image Qualities Between f-FOV and rFOV Diffusion-Weighted Imaging

\begin{tabular}{llll}
\hline Image parameter & f-FOV & r-FOV & $P$ value \\
\hline SNR & $40.75 \pm 10.30$ & $22.34 \pm 5.90$ & $<0.001$ \\
CNR & $5.24 \pm 1.29$ & $10.23 \pm 2.92$ & $<0.001$ \\
Sharpness & $2.64 \pm 0.59$ & $3.43 \pm 0.47$ & $<0.001$ \\
Distortion & $2.59 \pm 0.54$ & $3.35 \pm 0.61$ & $<0.001$ \\
Artifacts & $2.75 \pm 0.54$ & $3.66 \pm 0.46$ & $<0.001$ \\
Lesion conspicuity & $2.48 \pm 0.62$ & $3.39 \pm 0.55$ & $<0.001$ \\
Total subjective IQ & $10.41 \pm 1.11$ & $13.84 \pm 1.07$ & $<0.001$
\end{tabular}

Data are means \pm standard deviations (averages between two readers)

DWI and it has a long readout time and a narrow bandwidth in phase encoding direction. SSEPI may cause image distortion, image blur and signal loss, it also contains bone, soft tissue, liquid, gas and other substances which make the field uneven, and artifacts will be produced easily because of the large range of full field-of-view (f-FOV) $[11,13,14]$. The r-FOV DWI technique in this study uses a 2D radiofrequency pulse to selectively excite the small volume of interest, such as gallbladder, thereby reducing number of baseline required for $\mathrm{K}$-space filling, thus reducing readout time, so that the deformation and artifacts of images can be reduced effectively $[14,15]$.

In this study, the r-FOV DWI sequence provided a significantly better image quality. CNR and subjective image quality scores of $\mathrm{r}$-FOV DWI sequence were significantly higher than those of f-FOV DWI, which are consistent with previous studies. Sapkota et al. ${ }^{9}$ demonstrated that r-FOV DWI image presented with higher resolution and significantly reduced distortion. Similar results were also noted in abdominal organs.
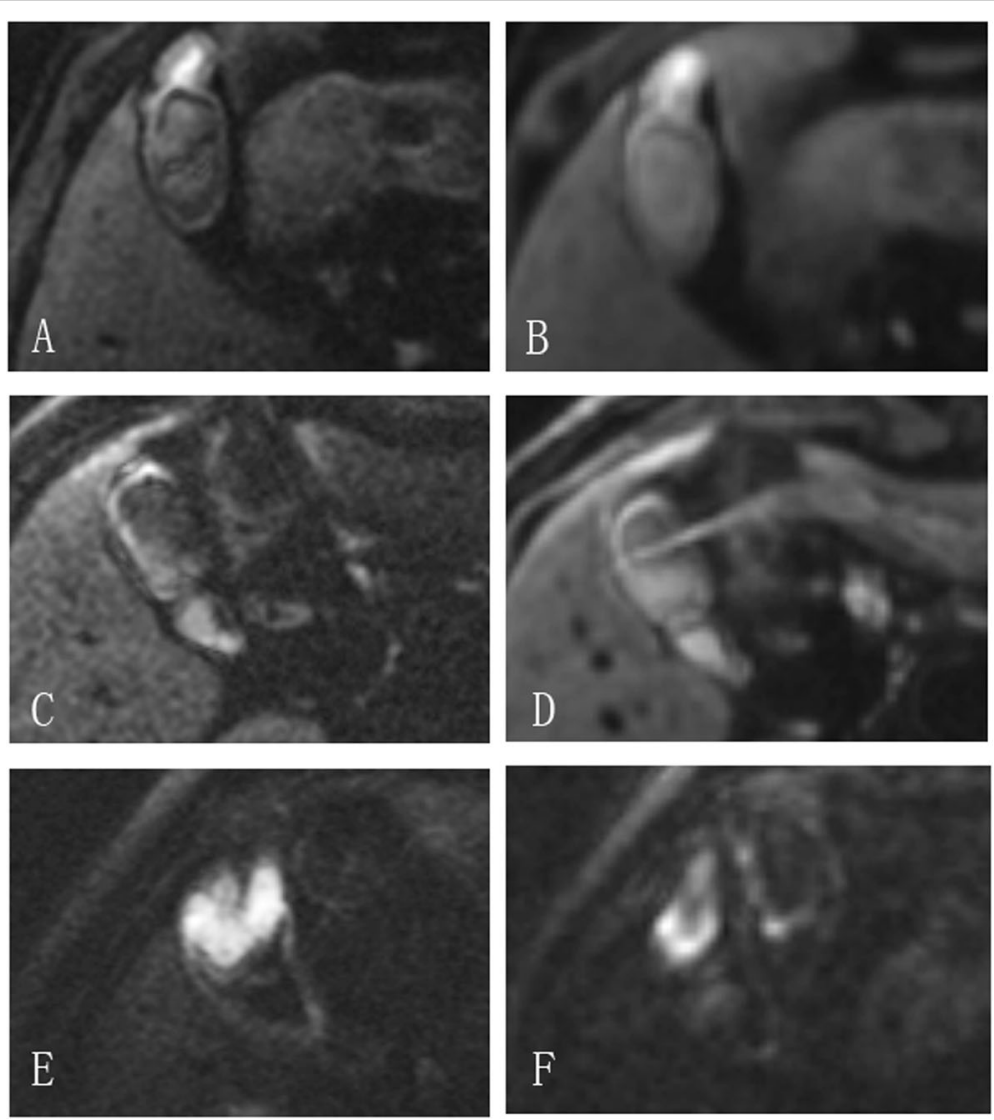

Fig. 2 Images for subjective quality assessment. a, b: Assessment of sharpness and lesion conspicuity (gallbladder adenomyomatosis). a: r-FOV DWI image shows the soft tissue signal at the bottom of the gallbladder with a sharp margin, and the lesion features are clearly demonstrated. Subjective image quality scores of sharpness and lesion conspicuity are both 3 . $\mathbf{b}$ : f-FOV DWI image shows the soft tissue signal at the bottom of the gallbladder with blurred edges and lower contrast comparing with Fig. A. The subjective image quality scores of sharpness and lesion conspicuity are both 2. c, d (gallbladder adenoma): Assessment of artifacts. c: r-FOV DWI image shows mild artifacts around the wall of the gallbladder. Subjective image quality score of artifacts is 4 . $\mathbf{d}$, f-FOV DWI image shows obvious artifacts. Subjective image quality score of artifacts is 3. $\mathbf{e}, \mathbf{f}$ (gallbladder adenocarcinoma): Assessment of distortion. $\mathbf{e}$ : r-FOV DWI image shows a diffusion-restricted lesion at the bottom of the gallbladder without distortion. Subjective image quality score of distortion is 4. $\mathbf{f}$ : f-FOV DWI image shows the edge of the lesion with obvious distortion. Subjective image quality score of distortion is 2 


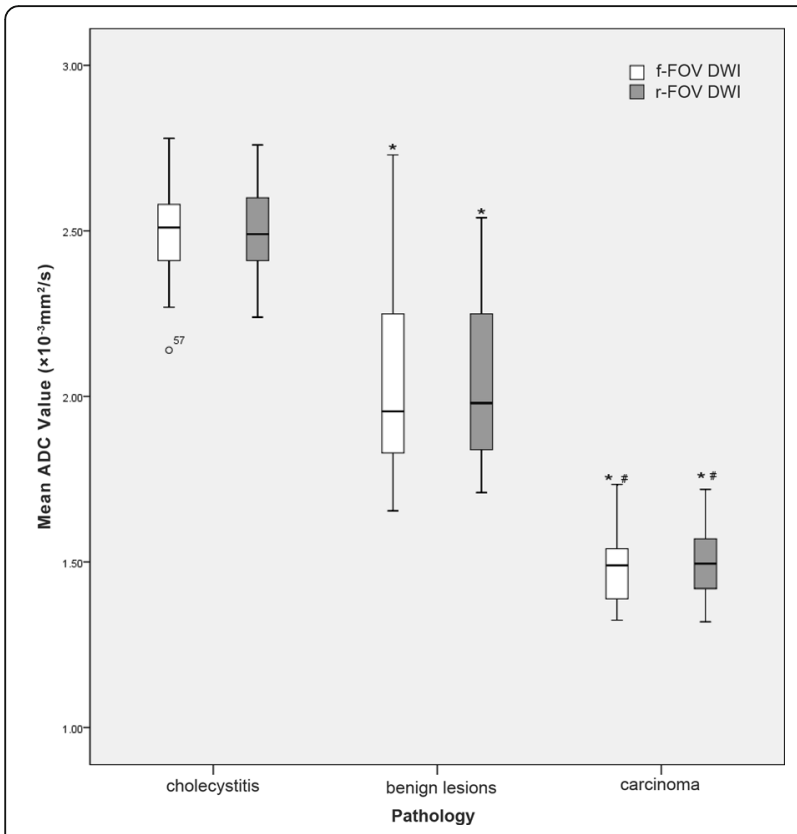

Fig. 3 Mean ADC values of both $f-F O V$ and $r$-FOV diffusion-weighted imaging related to pathological results. ${ }^{*} P<0.01$ versus the group of cholecystitis, ${ }^{\#} P<0.01$ versus the group of benign disease

Peng et al. [8] and Wang et al. [10] reported that image quality was significantly higher for r-FOV DWI images than the f-FOV DWI images in both rectal and urinary bladder tumors. Similar results were obtained in prostate tumor by Tamada et al [11] The r-FOW DWI sequence allowed the edge of the gallbladder wall and the structure of the tissue to be displayed more clearly, and it is easier to detect small lesions. However, the result of SNR was the opposite. Theoretically, SNR decreases as FOV reduces and image resolution increases, therefore, suitable reduced FOV, not smaller FOV is better at assessing gallbladder lesions [16].

In our study, there was no significant difference in the mean ADC values between two DWI sequences. Attenberger et al. [13] have shown that the ADC values of the prostate in different DWI sequences were not significantly different. Dong also had the same conclusion in

Table 4 Comparison of Apparent Diffusion Coefficient Values Between f-FOV and r-FOV Diffusion-Weighted Imaging Related to Pathological results

\begin{tabular}{llll}
\hline Pathology & \multicolumn{2}{l}{ ADC value $\left(\times 10^{-3} \mathrm{~mm}^{2} / \mathrm{s}\right)$} & v \\
\cline { 2 - 3 } & $\mathrm{f}$-FOV & r-FOV & \\
\hline 1 & $2.49 \pm 0.14$ & $2.50 \pm 0.14$ & 0.239 \\
2 & $2.05 \pm 0.29$ & $2.05 \pm 0.24$ & 0.974 \\
3 & $1.49 \pm 0.12$ & $1.50 \pm 0.13$ & 0.226 \\
Total & $2.02 \pm 0.46$ & $2.02 \pm 0.45$ & 0.625 \\
\hline
\end{tabular}

Data are means \pm standard deviations
Table 5 Spearman Rank Correlation Analysis of Apparent Diffusion Coefficient Values and Pathological Results

\begin{tabular}{lll}
\hline & $r$ value & $P$ value \\
\hline f-FOV & $-0.892(-0.933,-0.819)$ & $<0.001$ \\
$r$-FOC & $-0.913(-0.936,-0.857)$ & $<0.001$ \\
\hline
\end{tabular}

Data in parentheses are $95 \%$ confidence intervals

breast [16]. Our results are consistent with above results. However, the conclusion obtained in thyroid gland, lesion and muscle in the head and neck was totally different $[17,18]$. The reason for the different ADC values may be the lower resolution and poorer image quality of f-FOV DWI image, which affected by partial volume effect and magnetic sensitivity artifact when measuring the $\mathrm{ADC}$ value in the region of interest. The r-FOV DWI images have higher resolution, better fat saturation and fewer artifacts, thus the ADC values measured by $r$-FOV DWI is more closer to the genuine $\mathrm{ADC}$ value of the tissue.

In addition, the ADC value is affected by other factors, such as blood flow perfusion, b values of DWI and so on. The blood flow-rich organ is affected by blood flow, resulting error in the measurement of ADC values [19]. In our clinical protocol for abdominal DWI, the b-value was optimized to be $800 \mathrm{~s} / \mathrm{mm}^{2}$ based on our experience, so we chose a b-value of $800 \mathrm{~s} / \mathrm{mm}^{2}$ in $\mathrm{r}$-FOV DWI in order to compare these results with those in f-FOV DWI. The blood perfusion of the gallbladder was not abundant, thus, the influence of blood perfusion was avoided. Also, we selected the region of tissue with uniform signal instead of the part with artifacts and deformation while drawing the ROI, and the area of ROI remained the same in the two DWI sequences, which may be the possible reason that the difference between the measured $\mathrm{ADC}$ values in the two DWI sequences was not statistical significant.

Furthermore, our study indicated r-FOV DWI can detect the gallbladder tumor among gallbladder lesions. The mean ADC values of malignant tumor of gallbladder (group 3) was significantly lower than that of benign lesions of gallbladder (group 2) and cholecystitis (group 1), the mean ADC values of benign lesions of gallbladder (group 2) was significantly lower than that of cholecystitis (group 1). The pathological results include gallbladder adenoma and gallbladder polyps in group 2, they are both belonged to gallbladder polypoid-lesions (GPs), which have been considered as premalignant lesions of gallbladder, studies showed that $15.3 \%$ of GPs lesser than $10 \mathrm{~mm}$ in size were malignant, while the lesion was greater than 10 $\mathrm{mm}$, the malignant rate was increased [1, 20]. Therefore, the malignant trend of these three groups in increasing as the group number increases (in other words, the 
malignancy of group 1 was the lowest while group 3 was the highest). A significant tendency of a negative correlation between the mean ADC values and the pathological results was observed in both DWI sequences.

Our results are consistent with several prior studies. Kitazume et al. [5] demonstrated that the ADC value of malignant lesions was significantly lower than that of benign ones. Furthermore, Yoshioka et al. [21] showed that the ADC value was useful for differential diagnosis of inflammatory diseases, adenoma and cancer of the gallbladder. DWI reflects the random diffusion motion of water molecules, which are affected by cell density, microcirculation and histological composition, apparent diffusion coefficient (ADC) derived from DWI can be treated as a quantitative parameter [12]. Cancer cells in gallbladder proliferated rapidly, which leaded to the increasing of cell density, besides, the organelles, intracellular matrix and soluble macromolecules restricted the diffusion of water molecules, then the ADC values declined [21].

There are several limitations in this study. Firstly, the number of cases enrolled was relatively small, which could have resulted in selective bias of measured values. Secondly, the ROI drawing was based on the largest area of the lesion for adjacent three sections, which may not reflect the whole information of lesions, the whole volume measurement should be considered for further studies. Furthermore, benign gallbladder lesions (group 2) were not subdivided according to the specific pathological results. Further cohort studies should be performed with larger sample size and subdivision of pathological results to ensure the veracity and reliability.

\section{Conclusion}

The image quality of r-FOV DWI was significantly higher than that of f-FOV DWI. There was no significant difference in the mean ADC values between these two DWI sequences, and r-FOV DWI is a good tool to diagnose the gallbladder carcinoma.

\section{Abbreviations \\ ADC: Apparent diffusion coefficient; r-FOV DWl: Reduced field-of-view diffusion-weight imaging; f-FOV DWI : Full field-of-view diffusion-weight im- aging; DWI: Diffusion weighted imaging; SS-EPI: Single-shot echo-planar im- aging; MRI: Magnetic resonance imaging; PACS: Picture archiving and communication system; SNR: Signal-to-noise ratio; CNR: Cintrast-to-noise.; ROI: Region of interest}

\section{Acknowledgments}

This work was supported by the National Natural Science Foundation of China (No, 81771801, 81571642, 81701657, 81801695).

\section{Authors' contributions}

All authors reviewed the final manuscript prior to submission. All authors contributed to concept design, analysis and manuscript review. Sisi Wu and Chuou Xu performed quantitative analysis and review the MR scans, and authored the first draft of the manuscript. All authors read and approved the final manuscript.

\section{Funding}

This work was supported by the National Natural Science Foundation of China (No, 81771801, 81571642, 81701657, 81801695).

\section{Availability of data and materials}

Not applicable.

\section{Ethics approval and consent to participate}

The Ethics Committee of Tongji Hospital of Huazhong University of Science and Technology approved this study, and all patients provided written informed consent before their inclusion.

\section{Consent for publication}

The Authors declare that the paper is being submitted for consideration for publication in Cancer Imaging, the content has not been published or submitted for publication elsewhere. All authors have contributed significantly, and that all authors are in agreement with the content of the manuscript.

\section{Competing interests}

The authors declare that they have no competing interest.

Received: 7 January 2020 Accepted: 1 July 2020

Published online: 17 July 2020

References

1. Bhatt NR, Gillis A, Smoothey CO, et al. Evidence based management of polyps of the gall bladder: a systematic review of the risk factors of malignancy. Surgeon. 2016;14:278-86.

2. Hickman L, Contreras C. Gallbladder Cancer: diagnosis, surgical management, and adjuvant therapies. Surg Clin North Am. 2019;99:337-55.

3. Ratanaprasatporn L, Uyeda JW, Wortman JR, et al. Multimodality imaging, including dual-energy $C T$, in the evaluation of gallbladder disease. RADIOGRAPHICS. 2018;38:75-89.

4. Min JH, Kang TW, Cha Dl, et al. Apparent diffusion coefficient as a potential marker for tumour differentiation, staging and long-term clinical outcomes in gallbladder cancer. Eur Radiol. 2019;29:411-21.

5. Kitazume Y, Taura S, Nakaminato S, et al. Diffusion-weighted magnetic resonance imaging to differentiate malignant from benign gallbladder disorders. Eur J Radiol. 2016;85:864-73.

6. Kim SJ, Lee JM, Lee ES, et al. Preoperative staging of gallbladder carcinoma using biliary MR imaging. J Magn Reson Imaging. 2015;41:314-21.

7. Lee NK, Kim S, Moon Jl, et al. Diffusion-weighted magnetic resonance imaging of gallbladder adenocarcinoma: analysis with emphasis on histologic grade. Clin Imaging. 2016;40:345-51

8. $\quad$ Peng $Y, L i ~ Z$, Tang H, et al. Comparison of reduced field-of-view diffusionweighted imaging (DWI) and conventional DWI techniques in the assessment of rectal carcinoma at 3.0T: Image quality and histological T staging. J Magn Reson Imaging. 2018;47:967-75.

9. Sapkota N, Shi X, Shah LM, et al. Two-dimensional single-shot diffusionweighted stimulated EPI with reduced FOV for ultrahigh-b radial diffusionweighted imaging of spinal cord. Magn Reson Med. 2017;77:2167-73.

10. Wang Y, Li Z, Meng X, et al. Nonmuscle-invasive and Muscle-invasive Urinary Bladder Cancer: Image Quality and Clinical Value of Reduced Fieldof-view Versus Conventional Single-shot Echo-planar Imaging DWI. Medicine (Baltimore). 2016;95:e2951.

11. Tamada T, Ream JM, Doshi AM, et al. Reduced field-of-view diffusionweighted magnetic resonance imaging of the prostate at 3 tesla: comparison with standard Echo-planar imaging technique for image quality and tumor assessment. J Comput Assist Tomogr. 2017;41:949-56.

12. Taffel MT, Johnson EJ, Chandarana H. Diffusion quantification in body imaging. Top Magn Reson Imaging. 2017;26:243-9.

13. Attenberger UI, Rathmann N, Sertdemir M, et al. Small field-of-view singleshot EPI-DWI of the prostate: evaluation of spatially-tailored twodimensional radiofrequency excitation pulses. Z Med Phys. 2016;26:168-76.

14. Koh DM, Lee JM, Bittencourt LK, et al. Body diffusion-weighted MR imaging in oncology: imaging at 3 T. Magn Reson Imaging Clin N Am. 2016:24:31-44.

15. Ma C, Li YJ, Pan CS, et al. High resolution diffusion weighted magnetic resonance imaging of the pancreas using reduced field of view single-shot echo-planar imaging at 3 T. Magn Reson Imaging. 2014;32:125-31. 
16. Dong H, Li Y, Yu K, et al. Comparison of image quality and application values on different field-of-view diffusion-weighted imaging of breast cancer. Acta Radiol. 2016;57:19-24.

17. Lu Y, Hatzoglou V, Banerjee $S$, et al. Repeatability investigation of reduced field-of-view diffusion-weighted magnetic resonance imaging on thyroid glands. J Comput Assist Tomogr. 2015;39:334-9.

18. Vidiri A, Minosse S, Piludu F, et al. Feasibility study of reduced field of view diffusion-weighted magnetic resonance imaging in head and neck tumors Acta Radiol. 2017:58:292-300

19. Heusch P, Wittsack HJ, Kropil P, et al. Impact of blood flow on diffusion coefficients of the human kidney: a time-resolved ECG-triggered diffusiontensor imaging (DTI) study at 3T. J Magn Reson Imaging. 2013;37:233-6.

20. $\mathrm{Xu} \mathrm{A}, \mathrm{Hu} \mathrm{H}$. The gallbladder polypoid-lesions conundrum: moving forward with controversy by looking back. Expert Rev Gastroenterol Hepatol. 2017; 11:1071-80.

21. Yoshioka M, Watanabe G, Uchinami H, et al. Diffusion-weighted MRI for differential diagnosis in gallbladder lesions with special reference to ADC cut-off values. Hepatogastroenterology. 2013;60:692-8.

\section{Publisher's Note}

Springer Nature remains neutral with regard to jurisdictional claims in published maps and institutional affiliations.

Ready to submit your research? Choose BMC and benefit from:

- fast, convenient online submission

- thorough peer review by experienced researchers in your field

- rapid publication on acceptance

- support for research data, including large and complex data types

- gold Open Access which fosters wider collaboration and increased citations

- maximum visibility for your research: over $100 \mathrm{M}$ website views per year

At BMC, research is always in progress.

Learn more biomedcentral.com/submissions 